Co-morbidities, n (\%)

Hypertension, $\mathrm{n}(\%)$

Hypothyroidism, n (\%)

Type 2 Diabetes Mellitus, $\mathrm{n}(\%)$

Rheumatological diseases, $\mathrm{n}(\%)$

Cardiac disease, $\mathrm{n}(\%)$

Malignancies, $\mathrm{n}(\%)$

Additional medications (number of patients,

$\%)$

$\begin{array}{ccc}12(19.6) & 13(33.33) & \text { NS } \\ 12(19,6) & 4(10,2) & \text { NS } \\ 5(8.2) & 5(12.82) & \text { NS } \\ 5(8,2) & 3(7,7) & \text { NS } \\ 3(4,9) & 2(5,1) & \text { NS } \\ 2(3,2) & 2(5.13) & \text { NS } \\ 36(61) & 29(74.3) & \text { NS }\end{array}$

Conclusion: According to our preliminary data the majority of the patients continue to take colchicine after age of 40 . However the frequency of FMF attacks as well as daily colchicine dose decrease as the patients get older. With well designed trials stopping colchicine treatment may be considered in a subgroup of patients after 50 years of age. Approximately $3 / 4$ of the FMF population over 40 years of age has a comorbidity that necessitates additional medications which underlines the need for special attention.

Disclosure of Interests: None declared

DOI: 10.1136/annrheumdis-2019-eular.3892

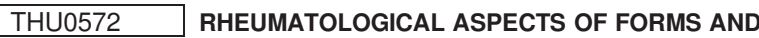 VARIANTS OF PANNICULITIS}

Olga Egorova ${ }^{1}$, Boris Belov ${ }^{1}$, Svetlana Glukhova ${ }^{1}, \mathrm{~S}$. Radenka-Lopovok ${ }^{2} .{ }^{1}$ V.A. Nasonova Research Institute of Rheumatology, Moscow, Russian Federation;

${ }^{2}$ 1FSBEIHE I.M. Sechenov Moscow State Medical University, Moscow, Russian Federation

Background: Panniculitis $(\mathrm{Pn})$ is a set of heterogeneous inflammatory diseases characterized by lesions of subcutaneous fat (SCF) and often accompanied with the defeat of the musculoskeletal system and internal organs. Depending on the etiology and histomorphological picture, $\mathrm{Pn}$ is divided into two forms: septal (SPn) and lobular panniculitis (LPn) with and without signs of vasculitis, which is reflected in the clinical picture of the disease. Often $\mathrm{Pn}$ is one of the symptoms of rheumatic diseases $(\mathrm{Rd})$, it can lead to late diagnosis of the underlying disease.

Objectives: to study the frequency of occurrence of forms and variants of $\mathrm{Pn}$ in rheumatological practice on the basis of long-term prospective observation

Methods: We examined 687 patients with $\mathrm{Pn}$ (613 women and 74 men, mean age $39.7 \pm 11.31$ and $41.2 \pm 12.57$ years, respectively) with the referral diagnosis of "Erythema Nodosum" (EN) or "Panniculitis", who were observed in the rheumatologic clinic in 2007-2017. In addition to general clinical examination, we determined serum concentrations of $\alpha-1$ antitrypsin, amylase, lipase, ferritin, creatine phosphokinase, conducted a CT scan of the chest organs, immunological, TB tests and pathological study of skin biopsy from the node.

Results: As a result of application of the developed diagnostic algorithm, 430 patients $(62.6 \%)$ were diagnosed with Spn and 249 patients $(36.2 \%)$ - with LPn. In 9 cases (1\%) (3 patients with SPn and 6 - with LPn), it was not possible to confirm the variant of $\mathrm{Pn}$, despite the comprehensive examination. In 8 patients $(1.1 \%)$ no $\mathrm{Pn}$ data were revealed, the following diagnoses were verified: 2 patients $(25 \%)$ - discoid lupus and lipodystrophy, 1 (12.5\%) - erysipelas, lichen, fixed erythema and keratoacanthoma. SPn in $94 \%$ of cases (400 patients) was presented by $\mathrm{EN}$, in $4 \%$ (18) - by superficial migrating thrombophlebitis, in single cases $(1-0 \%)$ - by eosinophilic fasciitis, skin nodular polyarteritis and scleroderma-Pn (2, 6 and 1 patients, respectively). In $28 \%$ of cases LPn was associated with systemic connective tissue lesions, in $27 \%$ with idiopathic lobular Pn (Weber - Christian panniculitis) ( $\mathrm{p}<0.002)$, in $32.92 \%$ with lipodermatosclerosis and in $14.28 \%$ with subcutaneous sarcoid. In a few cases Lpn was caused by lymphoproliferative $(2,88 \%)$ and cancer $(2,49 \%)$ diseases, calciphylaxis $(2,05 \%)$, erythema Bazin (1.64\%), cold Pn $(0.82 \%)$ and alpha 1 -antitrypsine failure $(0.41 \%)$

Conclusion: the results confirm that patients with SPn were found twice as often as with LPn. Prenosological Pn was mainly associated with sarcoidosis, EN and $\mathrm{Rd}$, which confirms the relevance of further research of $\mathrm{Pn}$.

Disclosure of Interests: None declared

DOI: 10.1136/annrheumdis-2019-eular.3730

\section{THU0573 \\ COMPLICATIONS AND ACTIVITY OF UVEITIS IN A MULTIDISCIPLINARY REFERENCE UNIT IN THE NORTH OF SPAIN}

Patricia Fanlo ${ }^{1}$, Henar Heras ${ }^{1}$, Alfredo Adan ${ }^{2}$, Gerard Espinosa ${ }^{3} .{ }^{1}$ Complejo Hospitalario de Navarra, Multidisciplinary Uveitis Unit, Pamplona, Spain; ${ }^{2}$ Hospital Clinic, Ophthalmology, Barcelona, Spain; ${ }^{3}$ Hospital Clinic, Autoimmune Systemic Disease Unit, Barcelona, Spain

Background: Uveitis, an intraocular inflammatory disease, is a significant cause of visual impairment. It is not known how many patients with uveitis will retain visual acuity and how many develop visual impairment or even blindness. The activity of uveitis, the use of topic and oral steroids could produce ocular complications.

Objectives: To describe the complications and the activity of the uveitis in a cohort of 500 patients diagnosed in one unit of reference in the North of Spain.

Methods: A prospective study of complications and the activity of the 500 adults with uveitis were evaluated in the multidisciplinary Uveitis Unit of Complejo Hospitalario de Navarra from January of 2010 to March of 2015. Uveitis activity and the type of complication to the year of followup were analysed. The variables were collected according to the terminology of the activity of the SUN (inactive, worsening, improvement and remission). To facilitate statistical analysis variables inactive and remission were grouped in a same term. A study of characterization of the evolution and worsening of the activity to the year of follow-up with age, sex, foreign, type of uveitis, etiology, laterality, treatments and complications The characterization was carried out through the SPAD 8 program and was carried out by contrast Chi square.

Results: We collected a total of 500 patients, $54.4 \%$ of patients remained inactive or in remission after 1 year of follow-up, $13.2 \%$ presented worsening of their activity and $32.45 \%$ had improved. A bivariate analysis was performed between the variable evolution per year and other variables. We díd'nt observe relationship between the evolution per year and age, sex, foreign, type of uveitis, etiology, laterality, topical etreatment, intravitreal treatment, biological treatment or surgical treatment. Relationship was found statistically significant between the evolution with the existence of complications $(p=0,0)$ and oral immunosuppressive treatment $(p=0,008)$. There was statistically significant relationship between the variable worsening with the existence of $\operatorname{complications}(p=0,0)$, topical eye treatment $(p=0,035)$, oral immunosuppressive treatment $(p=0$, $011)$, and not relationship with age was found $(p=0,8)$, sex $(p=0,35)$, foreign $(p=0,27)$, type of uveitis $(p=0,85)$, laterality $(p=0,6)$ etiology $(p=0,1)$, periocular treatment $(p=0,50)$, intravitreal $(0.56)$ and biologic treatment $(p=0,182) .65 \%$ of the patients did not show any com plication a year of follow-up and the most frequent complications were cataract $(10 \%)$, synechiae $(8 \%)$, macular edema $(5 \%)$, glaucoma $(3 \%)$, detachment of retina (3\%), epiretinal membrane $(2 \%)$ and other $(5 \%)$. Conclusion: $87 \%$ of the patients were in remission or improvement to the year of follow-up. The worsening of the uveitis is related with the existence of complications and oral immunosuppressive treatment.

\section{REFERENCES:}

[1] - D.A. Jabs, R.B. Nussenblatt, J.T. Rosenbaum, Standarization of uveitis nomenclature (SUN) Working Group Standarization of uveitis nomenclature for reporting clinical data. Results of the First International Workshop. Am J Ophthalmol. 2005; 140: 509-516

[2] - A. Rothova, M.S. Suttorp-van Schulten, W. Frits Tretters, A. Kijlstra. Causes and frequency of blindness in patients with intraocular inflammatory disease.Br J Ophthalmol.1996; 80: 332-336

[3] - Prieto-del-Cura M, González-Guijarro J. Complications of uveitis: prevalence and risk factors in a series of 398 cases.Arch Soc Esp Oftalmol. 2009;84:523-8

Disclosure of Interests: Patricia Fanlo Speakers bureau: Abvie, Henar Heras : None declared, Alfredo Adan : None declared, Gerard Espinosa - None declared

DOI: 10.1136/annrheumdis-2019-eular.1649 
THU0574

DOES THE USE OF CHOLCHICINE EFFECT COGNITIVE FUNCTIONS IN FAMILIAL MEDITERRANEAN FEVER? PRELIMINARY STUDY

Halise Hande Gezer ${ }^{1,1}$, Ozge Devezer Uslu², Didem Erdem ${ }^{1}$, Sevtap Acer Kasman ${ }^{1}$, Mehmet Tuncay Duruöz ${ }^{1} .{ }^{1}$ Marmara University School of Medicine, Department of Physical Medicine and Rehabilitation, Rheumatology Division, Istanbul, Turkey, ${ }^{2}$ Institution of Clinical and Forensic Psychology, Clinical Psychology, Istanbul, Turkey

Background: In Familial Mediterranean Fever (FMF), although cognitive functions have been shown to be impaired in children and adolescents, it has been shown that colchicine can be preservative on the cognitive functions in patients who are on long term colchicine treatment.

Objectives: This study aimed to evaluate cognitive functions in adult patients with FMF and cognitive effects of colchicine use.

Methods: The study included patients who were diagnosed with FMF according to Tel-Hashomer criteria. The control group included patients with no other inflamatory or systemic disease. Clinical features such as disease duration, comorbid diseases, colchicine treatment duration and dosage, amyloidosis and chronic renal failure (CRF), FMF gene mutation and PRAS scoring for evaluation of disease activity were recorded. Pittsburg Sleep Quality Index (PSQI), Fatigue Severity Scale, FMF Quality of Life Scale and Beck Depression Scale were used to assess patents clinical situations. Cognitive measurements were evaluated under executivepropellent functions. KAS test was used for information processing and fluency skills; fruit-name test was used for the evaluation of focusing, concentration, and attention skills; animal counting test and The Montreal Cognitive Rating Scale (MOCA) which can evaluate different subunits (visuospatial/executive, naming, memory, attention, language, abstraction, delayed recall, and orientation) were used for the fluency and maintenance of attention. Different attention parameters were evaluated for patients and healthy individuals. These attention parameters were; focusing, eloborate, sustainability, ability to pay attention to two information at the same time.

Descriptive analysis was performed for all parameters. Mann-Whitney Utest and Spearman correlation coefficient were used to compare parameters. $P<0.05$ was accepted as statistically significant.

Results: The study included 24 (21 women, 3 men) patients with FMF and 10 ( 7 female, 3 male) age, sex and BMl matched healthy controls. The mean age of the patients and controls were 36.83 (SD:10.9) and 39.3(SD:8.6), respectively. No significant difference was found between FMF and healthy control groups regarding animal counting, KAS test, MOCA test, and subgroups. Only fruit-name counting test was decreased in the FMF group compared to the healthy controls $(p<0.05)$. As the duration of colchicine treatment was prolonged, a moderate positive correlation was found in KAS scores $(r=0.511)$ and MOCA naming scores $(r=0.445)$. In the FMF group, the number of attacks in the last three months and the sleep scores of Pittsburgh and depression scores had a moderate positively correlation $(r=0.496)$. Depression scores and quality of life scores were highly correlated $(r=0.631)$.

Conclusion: FMF patients attention parameters are impaired compared to the healthy controls. Information processing and fluency performance is increased in FMF patients with the duration of colchicine treatment that demonstrate the ability to categorize and fluent use of the information. Number of attacks are correlated with poor sleep quality and depression.

\section{REFERENCES:}

[1] Keskindemirci, G., et al., Does familial Mediterranean fever affect cognitive function in children? Electrophysiological preliminary study. Int J Neurosci, 2018. 128(1): p. 10-14

[2] Leibovitz, A., et al., Colchicine therapy and the cognitive status of elderly patients with familial Mediterranean fever. Isr Med Assoc J, 2006. 8(7): p. 469-72.

Disclosure of Interests: Halise Hande Gezer: None declared, Ozge Devezer Uslu: None declared, Didem Erdem: None declared, Sevtap Acer Kasman: None declared, Mehmet Tuncay Duruöz Grant/research support from: Abvie, Speakers bureau: Novartis, AMGEN, Abdi İbrahim, İlko DOI: 10.1136/annrheumdis-2019-eular.6994

\section{THU0575 \\ HYPERCOAGULABILITY AS A CAUSE OF THROMBOSIS IN BEHCEET'S SYNDROME: A SYSTEMATIC REVIEW} AND META ANALYSIS

Gul Guzelant Ozkose, Berna Yurttas, Sinem Nihal Esatoglu, Vedat Hamuryudan Hasan Yazici, Gulen Hatemi. Istanbul University-Cerrahpasa, Medical Faculty of Cerrahpasa, Internal Medicine, Division of Rheumatology, Istanbul, Turkey

Background: While thrombosis in Behçet's Syndrome (BS) is considered to be mainly caused by inflammation in the vessel wall, several prothrombotic factors have been studied with inconsistent results.

Objectives: We aimed to perform a systematic review of clinical studies investigating the thrombophilic factors in BS

Methods: The online database of PubMed was searched with the keyword "Behcet" in four languages (English, German, French and Turkish) from inception up to May 2018. Titles and/or abstracts of all studies were screened independently by two reviewers (GG and BY) for studies reporting on thrombosis, fibrinolysis, endothelial factors and comparing BS patients with and without thrombosis. Conflicts were solved by a third reviewer $(\mathrm{GH})$. The pooled odds ratios $(\mathrm{OR})$ with $95 \% \mathrm{Cl}$ were calculated for binary outcomes and standardized mean differences (MD) were calculated for continuous outcomes by using RevMan 5.3.

Results: Of 9937 articles, 9373 were excluded due to repetition and inappropriate study design after reviewing titles and abstracts. Full text review of the remaining 564 articles yielded 86 papers meeting our predetermined inclusion criteria.

Several factors such as protein C, protein S, active protein C resistance, anti-thrombin III, plasminogen, plasminogen activator inhibitor, fibrinogen, factor 7, factor 12, thrombin activatable fibrinolysis inhibitor, anticardiolipin antibodies, antiß2 Glycoprotein1 antibodies and methylenetetrahydrofolate reductase gene C677T mutation were not different in BS patients with thrombosis compared to those without thrombosis. On the other hand, vascular endothelial growth factor levels, P-selectin glycoprotein ligand-1, platelet-activating factor seemed to be more frequent in BS patients with thrombosis in the few studies reporting on these, including a small number of patients.

Among the 11 parameters with controversial results across studies, metaanalysis showed significantly higher homocysteine levels, higher factor 8 levels, more frequent Factor $V$ Leiden mutations and higher von Willebrand factor levels in BS patients with thrombosis, whereas the pooled difference was not significant for mean platelet volume, tissue plasminogen activator, prothrombin gene mutations, lupus anticoagulant, P-selectin level, erythrocyte aggregation and thrombomodulin level (Table).

Conclusion: Among the several prothrombotic factors that were studied in BS patients, factor $V$ Leiden mutation, high homocysteine levels, factor 8 levels and von Willebrand factor levels may be associated with thrombosis in BS. Studies investigating these factors together in a large number of patients together with appropriate controls are needed to confirm these results.

Table. Meta-analysis of studies with controversial results

\begin{tabular}{|c|c|c|c|c|}
\hline \multirow[t]{2}{*}{ Prothrombotic Factor } & \multirow{2}{*}{$\begin{array}{l}\text { Number of } \\
\text { studies }\end{array}$} & \multicolumn{2}{|c|}{ Number of Behçet's patients } & \multirow{2}{*}{$\begin{array}{c}\mathrm{MD} / \mathrm{OR} \\
(95 \% \mathrm{Cl})\end{array}$} \\
\hline & & $\begin{array}{l}\text { With } \\
\text { thrombosis }\end{array}$ & $\begin{array}{l}\text { Without } \\
\text { thrombosis }\end{array}$ & \\
\hline vWF (U/dl) & 3 & 37 & 93 & $\begin{array}{c}\text { MD: } 10.09 \\
(2.70 \\
17.47)\end{array}$ \\
\hline Factor V Leiden & 9 & 204 & 446 & $\begin{array}{c}\text { OR: } 2.17 \\
(1.32,3.57)\end{array}$ \\
\hline Homocysteine & 12 & 285 & 436 & $\begin{array}{c}\text { MD: } 1.26 \\
(0.86,1.66)\end{array}$ \\
\hline FVIII level & 3 & 53 & 128 & $\begin{array}{c}\text { MD: } 14.68 \\
(2.87 \\
26.49)\end{array}$ \\
\hline tPA & 5 & 103 & 200 & $\begin{array}{c}\text { MD: } 0.09 \\
(-0.47 \\
0.66)\end{array}$ \\
\hline Prothrombin mutation & 7 & 189 & 398 & $\begin{array}{c}\text { OR: } 1.63 \\
(0.85,3.13)\end{array}$ \\
\hline Mean platelet volume & 5 & 73 & 327 & $\begin{array}{c}\text { MD: } 0.13 \\
(-0.13, \\
0.39)\end{array}$ \\
\hline
\end{tabular}

